RFP-2062

lugust 3, 1973

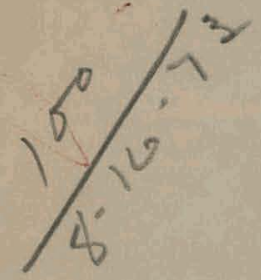

$$
22,84^{3}
$$

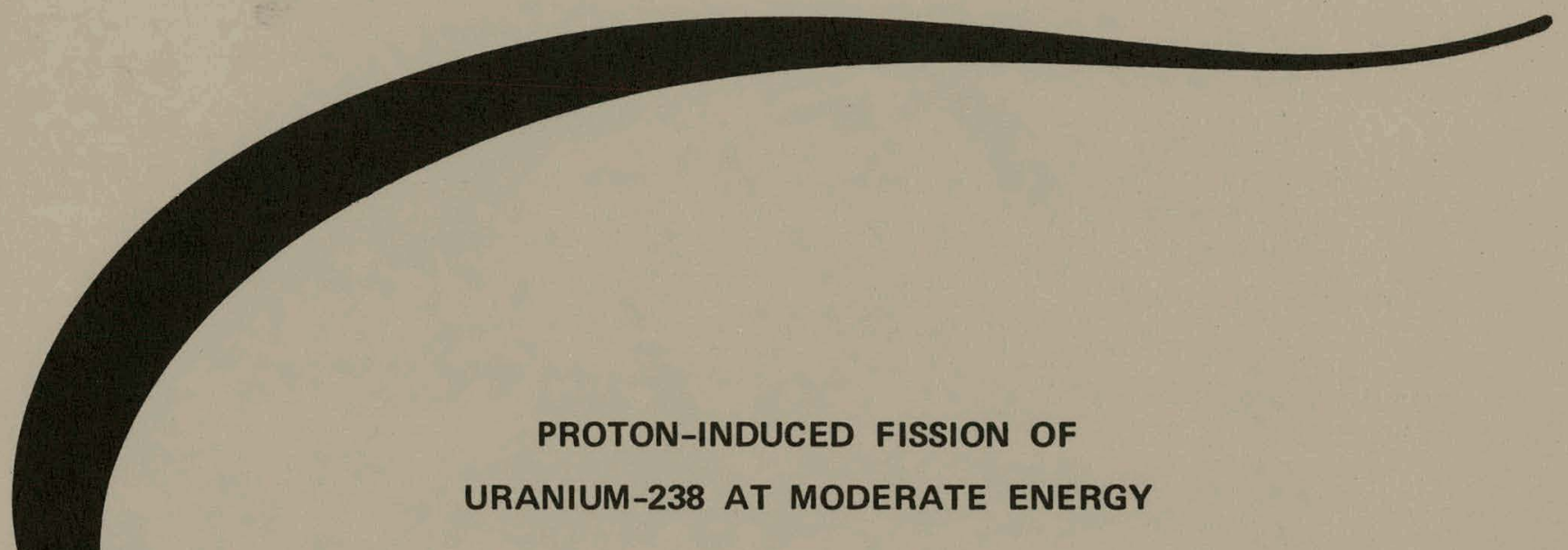

Larry D. Miller

Leo Yaffe

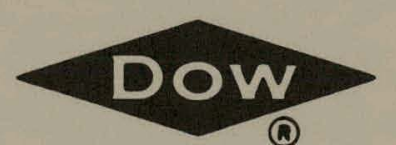

DOW CHEMICAL U.S.A.

ROCKY FLATS DIVISION

P. O. BOX 888

GOLDEN, COLORADO 80401

U.S. ATOMIC ENERGY COMMISSION CONTRACT AT(29-1)-1106 


\section{DISCLAIMER}

This report was prepared as an account of work sponsored by an agency of the United States Government. Neither the United States Government nor any agency Thereof, nor any of their employees, makes any warranty, express or implied, or assumes any legal liability or responsibility for the accuracy, completeness, or usefulness of any information, apparatus, product, or process disclosed, or represents that its use would not infringe privately owned rights. Reference herein to any specific commercial product, process, or service by trade name, trademark, manufacturer, or otherwise does not necessarily constitute or imply its endorsement, recommendation, or favoring by the United States Government or any agency thereof. The views and opinions of authors expressed herein do not necessarily state or reflect those of the United States Government or any agency thereof. 


\section{DISCLAIMER}

Portions of this document may be illegible in electronic image products. Images are produced from the best available original document. 


\section{LEGAL NOTICE}

This report was prepared as an account of work sponsured by the United States Government. Neither the United States nor the United States Atomic Energy Commission, nor any of their employees, nor any of their contractors, subcontractors, or their employees, makes any warranty, expressed or implied, or assumes any legal liability or responsibility for the accuracy, completeness or usefulness of any information, apparatus, product or process disclosed, or represents that its use would not infringe privately owned rights.

Printed in the United States of America

Available from the

National Technical Information Service

U. S. Department of Commerce

Springfield, Virginia 22151

Price: Printed Copy $\$ 4.00$ Microfiche $\$ 0.95$ 
Printed

August 3, 1973
RFP-2062

UC-34c-PHYSICS-NUCLEAR

TID-4500-R60

\section{PROTON-INDUCED FISSION OF \\ URANIUM-238 AT MODERATE ENERGY}

Larry D. Miller

Research and Ecology

HEALTH PHYSICS RESEARCH GROUP

\section{Leo Yaffe}

Radiochemistry Laboratory, Department of Chemistry McGill University Montreal, (Quebec) Canada

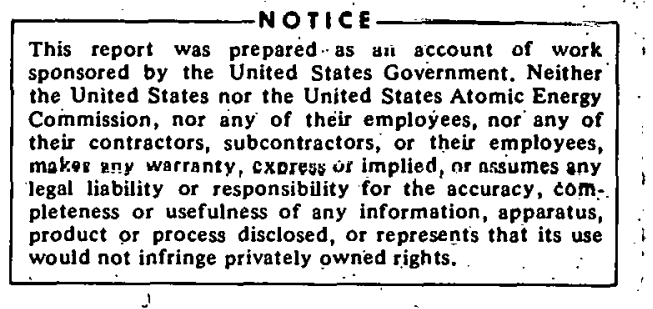

DOW CHEMICAL U.S.A. ROCKY FLATS DIVISION

P. O. BOX 888

GOLDEN, COLORADO 80401

Prepared under Contract AT(29-1)-1106

for the

Albuquerque Operations Office

U. S. Atomic Energy Commission

?ִ

\section{SUBJECT DESCRIP'IORS}

Fission

Radiochemistry

Proton Reactions

Antimony

Gamma Detection

Germanium-Lithium Drifted Detectors Isomers 


\section{CONTENTS}

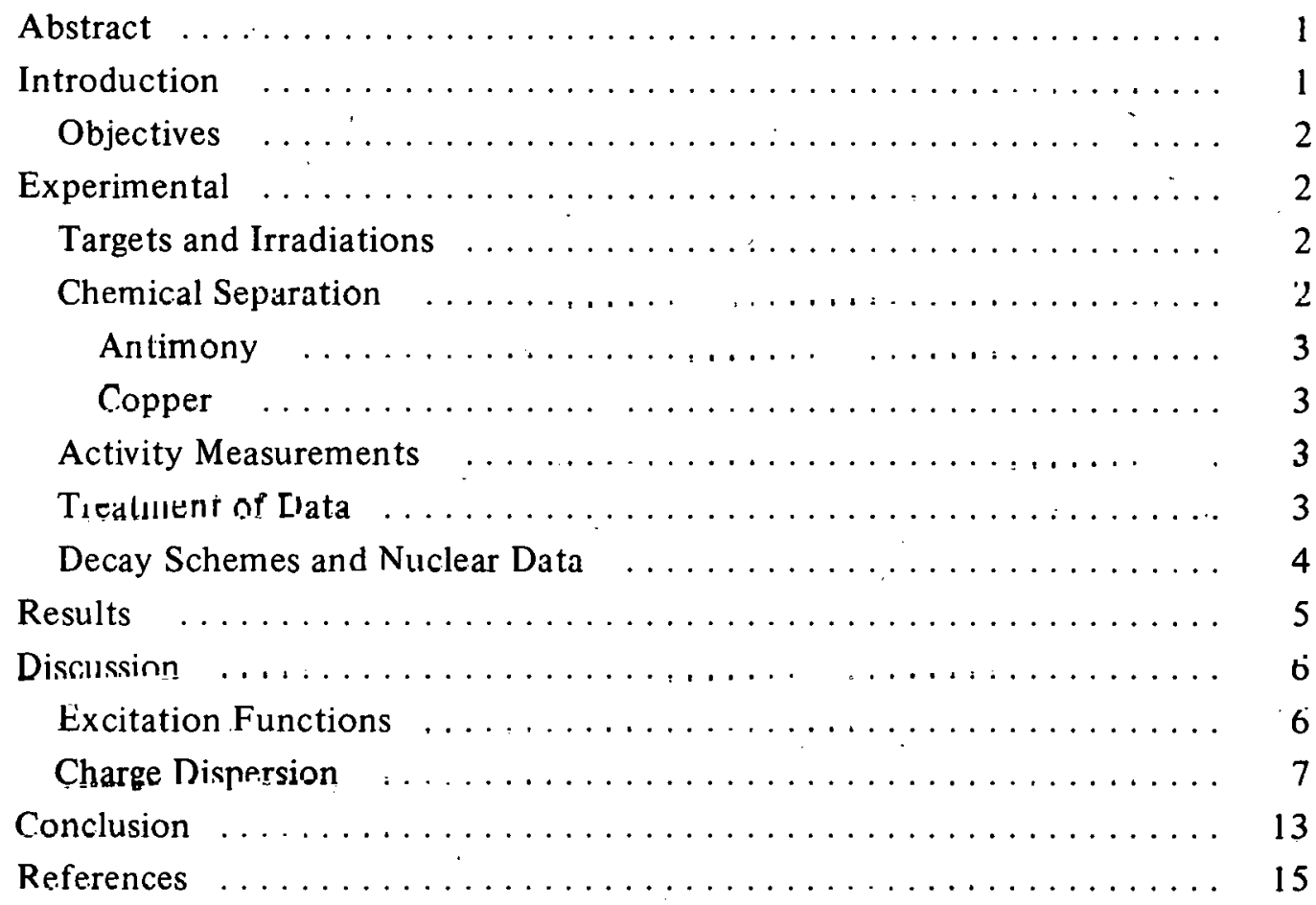




\title{
PROTON-INDUCED FISSION OF URANIUM-238 AT MODERATE ENERGY
}

\author{
Larry D. Miller and Leo Yaffe
}

\begin{abstract}
Independent and cumulative formation cross sections in the fission of uranium-238 $\left({ }^{238} \mathrm{U}\right)$ by protons of energy of 30 to 160 million elcctron volts $(\mathrm{MeV})$ have been determined for antimony isotopes of mass number 120 to 131 . Described are charge-dispersion curves $\left(Z_{p}=\right.$ probable charge) constructed and corrected for the variation of the neutron-to-proton ratio of the most probable fission product $N / Z_{p}$, as a function of mass (A). The charge-dispersion curves show an asymmetry and are computer-fitted by two overlapping Gaussians. If the curves could be correctly interpreted by the two-mode fission hypothesis, the ratios of asymmetric to symmetric fission at 50 and $160 \mathrm{MeV}$ would be 7.9 and 1.5 , respectively. The study is hased on a thesis submitted by Larry D. Miller in partial fulfillment of the requirements for the Doctor of Philosophy Degrce at the McGill University in Montreal, Canada. Financial assistance was given by the National Research Council of Canada.
\end{abstract}

\section{INTRODUCTION}

Many of the basic features of the distribution of nuclear charge in the thermal-neutron fission of uranium-235 $\left({ }^{235} \mathrm{U}\right)$ have been elucidated by the work of Wahl and his co-workers (1). ${ }^{1}$ Limited work was done on the problem of charge dispersion at higher energies before the work of Pate, Foster, and Yaffe (2) and that of Friedlander et al. (3). These authors studied isotopic distributions and found that, at proton energies below 380 million electron volts ( $\mathrm{MeV})$, a symmetric bell-shaped curve was obtained when the yields were plotted against the neutron-to-proton ratio $(\mathrm{N} / \mathrm{Z})$ of the fission product. These curves, though broader, are not unlike the isobaric curves, and may be utilized to extract information for comparisons with isobaric data. The most probahle charge, $Z_{p}$, determined from isotopic experiments, when assigned

\footnotetext{
${ }^{1}$ Numerals in parentheses relate to references at end of text.
}

to the mass at the peak of the curves (nonintegral) will be identical to the values of $Z_{p}$ obtained from isobaric curves. Furthermore it is possible to determine a relationship between the full-width at halfmaximum (FWHM) of the one curve to the other (4).

A fairly cxtensive body of data now exists for the proton-induced fission of uranium-238 $\left({ }^{238} \mathrm{U}\right)$ in the energy region 20 to $85 \mathrm{MeV}$. Davies and Yaffe (5) studied the dispersion of cesium isotopes, Parikh et al. (6) the dispersion in the mass range $\mathrm{A}=139$ to 143 , and Khan et al. (7) investigated yttrium isotopes in the mass range $A=90$ to 93 . In addition, a number of studies exist in these mass ranges with different fissile targets, such as plutonium-239 (8), uranium-233 (9), uranium-235 (10), thorium-232 (11), and neptunium-237 (12), conducted to determine the role of the neutron-toproton ratio of the target in determining the charge dispersion.

The following basic trends have been observed:

1. The peaks of the charge-dispersion curves shift towards beta stability with increasing bombarding energy. Mainly, the effect relates to increased neutron boil-off from the target nucleus.

2. The charge-dispersion curves broaden with increasing bombarding energy. This is caused by neutron evaporation from the target nucleus, the fission fragments, and the statistical nature of the neutron evaporation process.

3. A strong correlation appears to exist between the energy at which a certain product will have the greatest probability of being produced, the neutron-to-proton $(\mathrm{N} / \mathrm{Z}$ ) value, and the $\mathrm{N} / \mathrm{Z}$ value of the fissile target.

4. In the mass range 130 to 140 , from the variation of $\left(Z_{A}-Z_{p}\right)$, where $Z_{A}$ is the most stable 
charge for mass $A$, the value of $Z_{p}$ can be predicted from a knowledge of the value of $N / Z$ of the fissioning target $(12,13)$. No such evidence exists for the complementary light-mass products $(A=90$ to 100$)$. The effect has been ascribed to differences in neutron-emitting characteristics of the two fragments.

To date, no systematic correlation has been possible regarding the FWHM values of the charge-dispersion curves at medium bombarding energies. One reason may relate to lack of knowledge of the variation of peak position, $N / Z_{p}$, with mass number $A$. The assumption that the value of $N / Z_{p}$ is constant with $A$ is not correct as shown by Hogan and Sugarman (4). The assumption is held to be responsihle for the fact that the charge dispersion curves at energies around $400-\mathrm{MeV}$ bombarding energy are too broad when compared with those obtained from isobaric studies. Pappas and Hageb $\phi(14)$ have shown that at $170 \mathrm{MeV}, N / Z_{p}$ is constant with $A$ only in the narrow region near symmetric fission, $A=95$ to 115 . However, as will be shown later, plots of $N / Z_{p}$ against $A$ allow a correction for this variation.

If one corrects for the variation of chain yicld with mass, to take into account the slope of the massyield curve and the variation of $N / Z_{p}$ with $A$, the resulting isotopic dispersion may be compared with the isobaric charge-dispersion curve. This was done by Hogan and Sugarman (4) from the data of Pappas and Hageb $\phi(14)$ at $170 \mathrm{MeV}$.

\section{Objectives:}

The purpose of the present work is to extend the studies carried out in the laboratory at McGill University concerning the energy-dependence of chargedispersion curves. The present study in the antimiony region was chosen to fill the gap between the yttrium and cesium regions previously studied. This enables the determination of the shape of the function of $N / Z_{p}$ versus $A$, assumed to be constant in previous works. The data will also enable a correlation to be found between the widths of chargedispersion curves and the mass region under investigation. Of interest also will be to determine whether the data obtained would fall on the empirical curve obtained by Friedlander et al. (3), as extended by
Davies and Yaffe (5), wherein the energies at which the peaks of the excitation functions of the products obtained were plotted against the neutron-to-proton ratio of the product, resulting in a monotonic function.

\section{EXPERIMENTAL}

Targets and Irradiations:

The targets, consisting of a stack of foils described below and mounted on an aluminum target holder, were irradiated in the internal beam of the McGill University synchrocyclotron. The current varied between 1.0 and 1.8 microamperes $(\mu \mathrm{A})$ and the duration of irradiation varied between 10 to 20 minutes. Two irradiations were performed in the internal beam of the 150-inch synchrocyclotron at the Enrico Fermi Institute, University of Chicago, Chicago, Illinois, arranged by N. Sugarman. The energy range in the McGill University cyclotron used was from 30 to $85 \mathrm{MeV}$ and the energy spread was $\pm 2 \mathrm{MeV}$. A nominal.proton enorgy of 160 $\mathrm{MoV}$ was chusen for irradiations conducted at the University of Chicago.

The target foils of natural uranium varied in thickness from 0.005 to 0.012 centimeters $(\mathrm{cm})$. All were cut to approximately 1 by $2 \mathrm{~cm}$, with the exception of those in two experiments, in which the foils were trimmed to exactly 2.54 by $5.08 \mathrm{cms}$ with a special die. The target foil was protected against loss due to recoil by aluminum catcher foils $(0.0025 \mathrm{~cm})$ placed on either side.

The reactions used to monitor the proton beam were ${ }^{65} \mathrm{Cu}$ (p,pn) ${ }^{64} \mathrm{Cu}$ at proton energies up to $85 \mathrm{MeV}$ and ${ }^{27} \mathrm{Al}$ (p, 3pn) ${ }^{24} \mathrm{Na}$ at $160 \mathrm{MeV}$. The monitors, high purity copper or aluminum, consisted of a stack of three identical foils (to compensate for recoil losses) placed upstream from the target with respect to the proton beam. The center foil was used to determine the number of protons passing through the target.

\section{Chemical Separation:}

Immediately following the end of irradiation approximately 5 millimeters $(\mathrm{mm})$ of the leading edge of 
the target stack was sheared off for chemical processing and radiochemical assay. The uranium target foil, together with the aluminum catcher foils, was dissolved in a minimum volume of concentrated hydrochloric acid $(\mathrm{HCl})$, to which a minute amount of hydrofluoric acid (HF) and antimony carrier had been added. An aliquot of this solution was taken for antimony determination.

ANTIMONY (Sb)-The procedure used was a slight modification of the stibine-generation technique described by Greendale and Love (15). This is a rapid one-step procedure based on the Marsh test (16), but with improved chemical yield. The stibine was passed through a tube containing 8-mesh Drierite [ to remove contaminants, especially antimony iodide $\left(\mathrm{SbI}_{3}\right)$ ], decomposed by heat, and collected partly as an anlimony mirror on a quartz tube and the remainder as antimony metal on a sintered glass filter. The antimony was dissolved in $6 N \mathrm{HCl}$ saturated with bromine $\left(\mathrm{Br}_{2}\right)$, boiled to remove excess $\mathrm{Br}_{2}$ and reduced to $\mathrm{Sb}^{+3}$ with sodium hydrogensulfite $\left(\mathrm{NaHSO}_{3}\right)$. The solution was boiled to remove sulfur dioxide $\left(\mathrm{SO}_{2}\right)$ and antimony trisulfide $\left(\mathrm{Sb}_{2} \mathrm{~S}_{3}\right)$ was precipitated. The precipitate was filtered, washed, dried, and weighed to determine the chemical yield (usually of order of 70 percent).

When necessary to follow the decay of short-lived isotopes of antimony, or to isolate antimony quickly from its tin precursors, the relatively slow steps involving the antimony mirror and the sulfide precipitation were omitted. The stibine was passed directly into an aqueous solution of silver nitrate $\left(\mathrm{AgNO}_{3}\right)$. The resulting mixture of silver $(\mathrm{Ag})$, antimony, antimony oxide $\left(\mathrm{Sb}_{2} \mathrm{O}_{3}\right)$, and the compound, $\mathrm{Ag}_{3} \mathrm{Sb}$, was filtered, mounted, and the activity measured. To determine the chemical yields, the activily of shiclded ${ }^{126} \mathrm{Sb}$ in these, samples was compared to that in a second sample whose chemical yield was ascertained by the sulfide precipitation method described.

COPPER $(\mathrm{Cu})-$ The procedure was based on the elution technique of Kraus and Moore (17). The center copper foil was weighed, dissolved in '2milliliter ( $\mathrm{ml}$ ) concentrated $\mathrm{HCl}$ plus 4 drops 30 percent hydrogen peroxide $\left(\mathrm{H}_{2} \mathrm{O}_{2}\right)$, then the solution was evaporated to dryness, the residue taken up in $2-\mathrm{ml} 4.5 \mathrm{~N} \mathrm{HCl}$ and absorbed on an anionexchange resin column (pre-treated with $4.5 \mathrm{~N} \mathrm{HCl}$ ). Copper was eluted with 3 -column volumes $1.5 \mathrm{~N}$ $\mathrm{HCl}$, the middle fraction being taken for copper determination. The eluant was diluted to $0.5 \mathrm{~N}$ $\mathrm{HCl}$, the copper reduced to cuprous ion by boiling with $\mathrm{NaHSO}_{3}$ and copper thiocyanate (CuSCN) was precipitated with ammonium thiocyanate $\left(\mathrm{NII}_{4} \mathrm{SCN}\right)$. The precipitate was filtered, washed, weighed, and mounted for activity measurements.

\section{Activity Measurements:}

The gamma-ray $(\gamma)$ spectra were measured with a germanium-lithium [Ge (Li)] detector of 2.0-cubic centimeter $\left(\mathrm{cm}^{3}\right)$ active volume coupled to a 1600 channel analyzer. The detector resolution was 4.5 kiloelectron volt ( $\mathrm{keV}$ ) full-width at half-maximum for the cesium-137 $\left({ }^{137} \mathrm{Cs}\right) 662-\mathrm{keV} \gamma$-ray. The detector resolution was sufficient to allow direct determination of several fission products, (e.g., ${ }^{125} \mathrm{Sb}$ ) without prior chemical separation...

\section{Treatment of Data:}

The formation cross sections were calculated using the monitor cross sections of Meghir ( $\overline{1} \overline{8})$ and Cumming (19) for copper and aluminum, respectively. The data used are shown in Table $\mathbf{I}$.

TABLE I. Monitor Reaction Cross Sections.

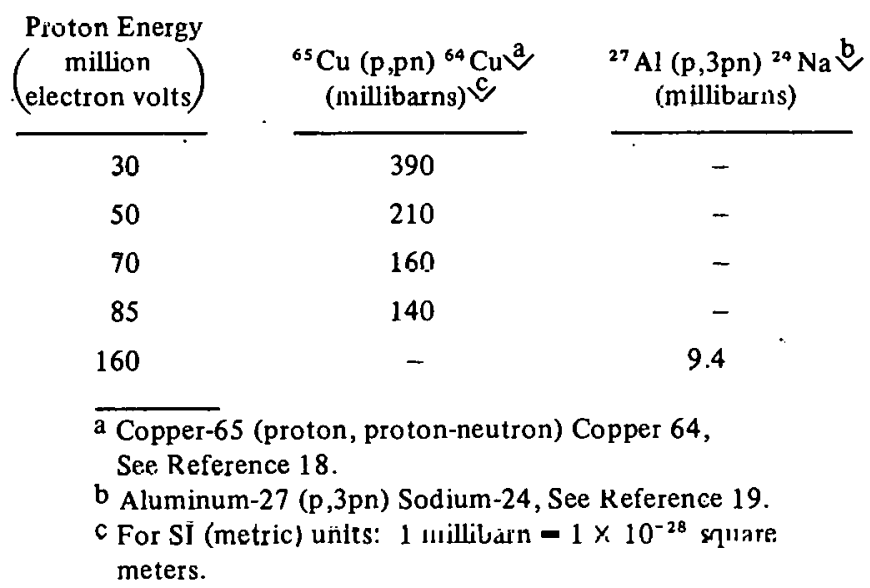


The methods of calculation used are similar to those of Friedlander et al. (3) and will not be repeated. The calculation in the case of the independent cross section of a shielded nuclide or the cumulative cross section of a nuclide with shortlived parents is perfectly straightforward. When the parent decay made a substantial contribution to the activity of the daughter before parentdaughter separation, the timed-separation technique was used with the appropriate growth and decay factors.

\section{Decay Schemes and Nuclear Data:}

The nuclear data used are given in Table II. Since most of the antimony isotopes measured are oddodd nuclei, close to a shell edge, considerable isomerism exists. In all cases, only the longerlived isomers were measured, so that knowledge of the isomer ratio is required to determine the cross section $(\sigma)$. These ratios have been determined by
Hageb $\phi(20)$ for proton-bombarding energies bctween 159 and $570 \mathrm{MeV}$. Those for ${ }^{124} \mathrm{Sb}$ and ${ }^{126} \mathrm{Sb}$ are constant and can be extrapolated to the energy region of interest. The isomer ratio for ${ }^{120} \mathrm{Sb}$ is not constant and no extrapolation has been made below $160 \mathrm{MeV}$.

The cross section for the 60.4-day isomer of ${ }^{124} \mathrm{Sb}$ was converted to the total nuclidic yield by assuming that the isomer ratio of $\sigma_{\mathrm{g}} / \sigma_{\mathrm{m}}=15.8$, (where $\mathrm{g}=$ ground state and $\mathrm{m}=$ metastable state) determined at higher energies, is unchanged down to $30 \mathrm{MeV}$ bombarding energy. Similarly, if one takes Hageb $\phi$ 's value, the isomer ratio $\sigma_{\mathrm{g}} / \sigma_{\mathrm{m}}=$ $4.4 \mathrm{for}{ }^{126} \mathrm{Sb}$, and the branching of the isomeric transition is 14 percent. The total cruss section is 1.19 times the experimentally-determined ground state (9). Aras and Gordon (21) calculated the isomer ratio for ${ }^{126} \mathrm{Sb}$ produced in thermal-neutron fission of ${ }^{235} \mathrm{U}$ and obtained a value of 4.8 , in good agreement with that of Hageb $\phi$ for the ${ }^{238} U(p, f)$ ${ }^{126} \mathrm{Sb}$ reaction $(\mathrm{p}=$ proton, $\mathrm{f}=$ fission $)$.

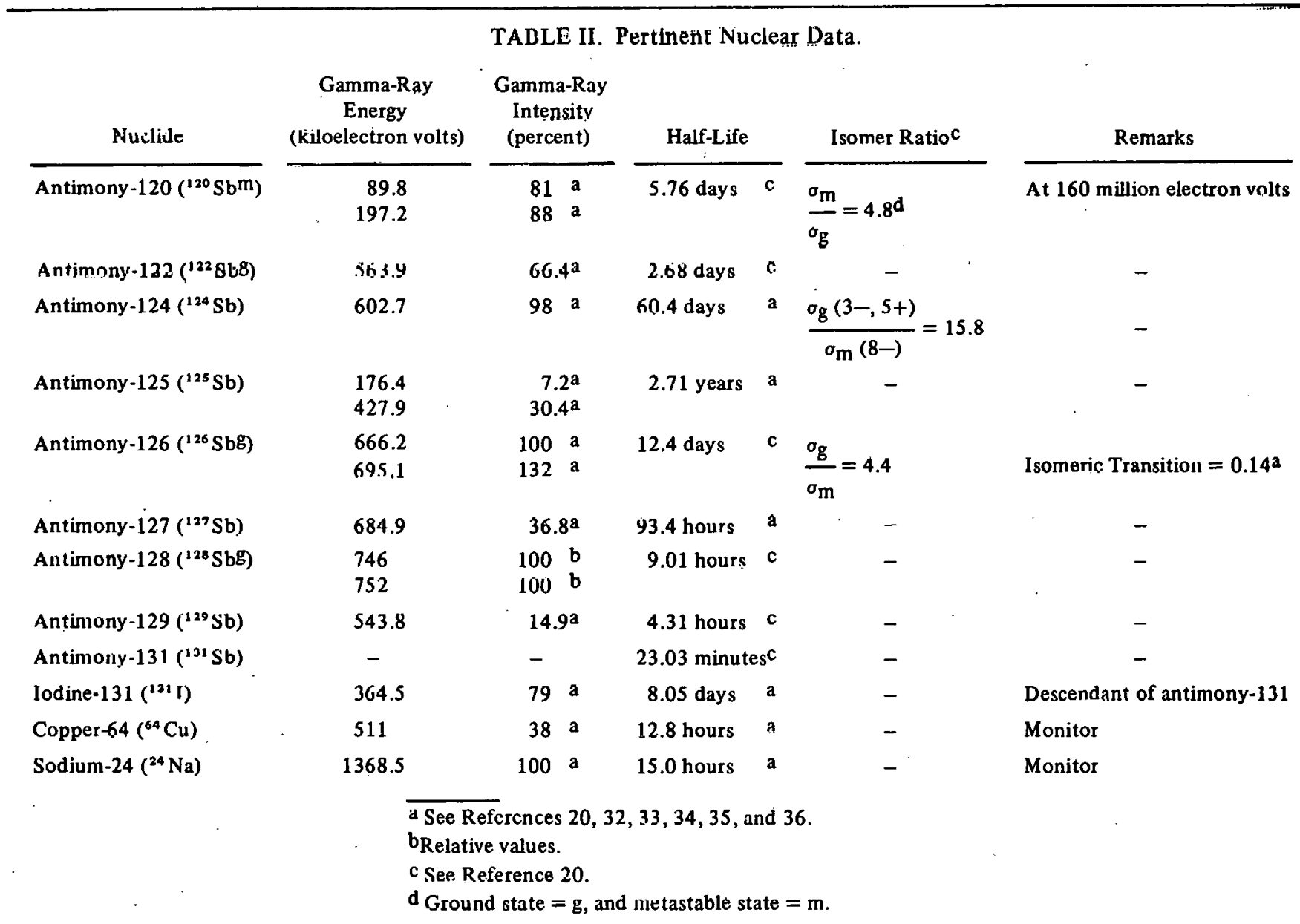




\section{RÉSÜLTTS}

The cross sections are shown in Table III. The small contribution (less than 10 percent) of the short-lived tin ( $\mathrm{Sn}$ ) isomers to the yield of ${ }^{125} \mathrm{Sb}$ and ${ }^{127} \mathrm{Sb}$ has been neglected. The data given in Table III were extended by those of Panontin and Sugarman (22) at $450 \mathrm{MeV}$ and Hageb $\phi(20)$ at $570 \mathrm{MeV}$ and 18.2 gigaelectron volts (GeV) to form the excitation functions shown in Figure 1 and the charge dispersion curves shown in Figures 2 and 3.

Figure 2 is a typical curve at $70-\mathrm{MeV}$ bombarding energy. It has been constructed by least squares fitting of a Gaussian constrained, so that the independent yields of the isobars for mass- 127 sum to the experimentally-determined cumulative yield of ${ }^{127} \mathrm{Sb}$. The intensity of the $\gamma$-rays listed in Table II for ${ }^{128} \mathrm{Sb}^{\mathrm{g}}$ was assumed to be 100 percent, thus providing a limit for the yield. This value has been plotted in Figure 2. From the charge-dispersion curves, interpolation is possible of the yields of ${ }^{128} \mathrm{Sb}$. Thus an isomer ratio will be obtained which is correct only relatively at the various energies. This ratio was found to be constant between $\mathbf{3 0}$ i and $85 \mathrm{MeV}$.
Figure 3 is a composite giving charge-dispersion curves from 30 to $160 \mathrm{MeV}$. Previous workers $(5,6,7)$ had used hand-drawn curves, assuming no geometric function, with only the constraint that the independent yields of the isobars, read from the curves, must equal to the sum of the cumulative yields of the isobar, experimentally determined, to which the independently-formed isobars must lead. The curves in this work were assumed to be parabolic in nature and the data fitted by a leastsquares computer program. The procedure, while less arduous, leads essentially to the same results.

The choice of a parabola was made since the thermal-neutron data are approximated by a Gaussian function (1). The expression for a Gaussian distribution is:

$$
P(Z)=\frac{1}{\sqrt{c \pi}} \exp \left[\frac{-\left(Z-Z_{p}\right)^{2}}{c}\right] \quad \cdots
$$

In Equation 1,

$$
\begin{gathered}
P(Z) \text { - fractional independent yield } \\
c \text { - constant for width of curve }
\end{gathered}
$$
'

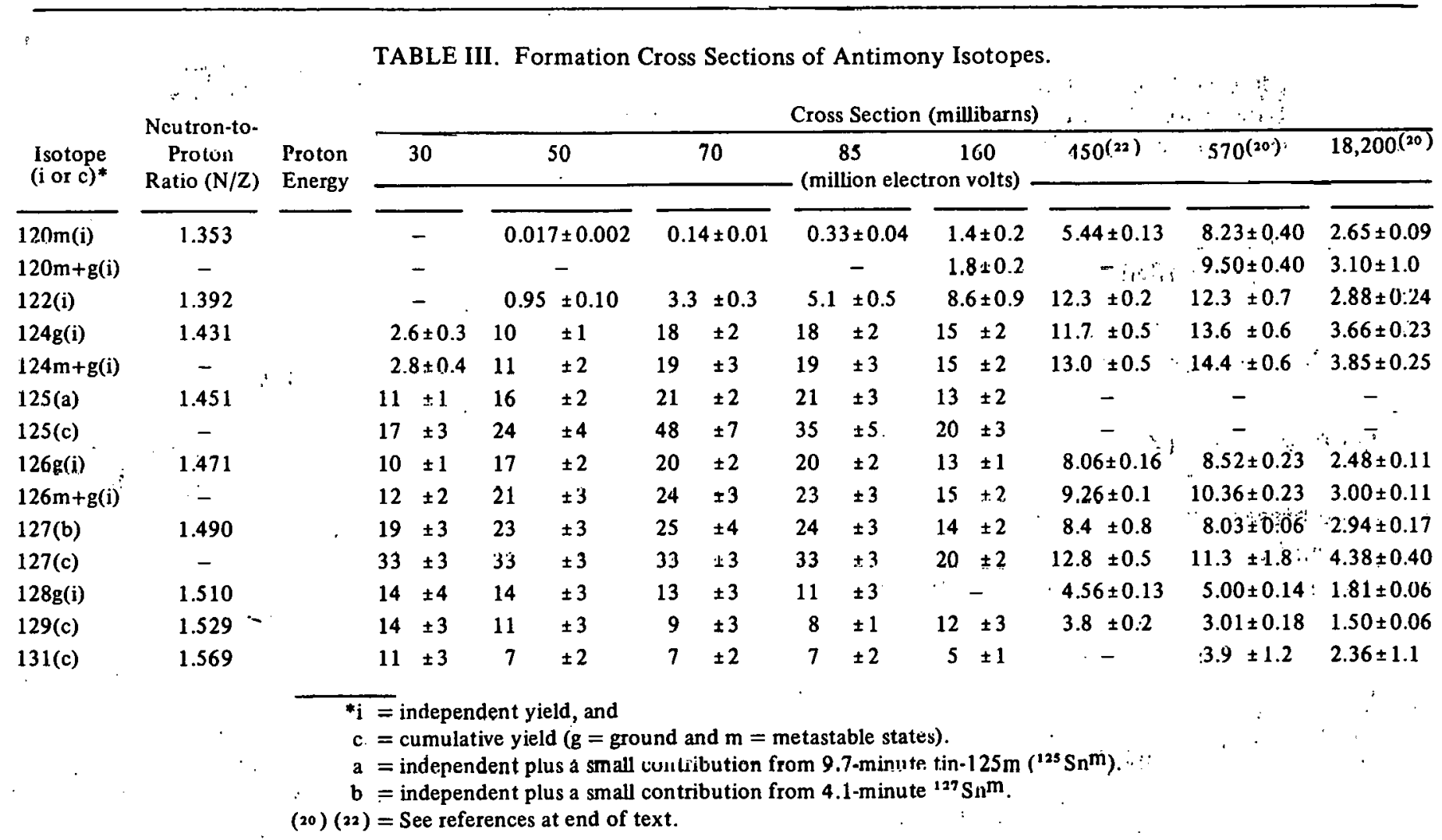




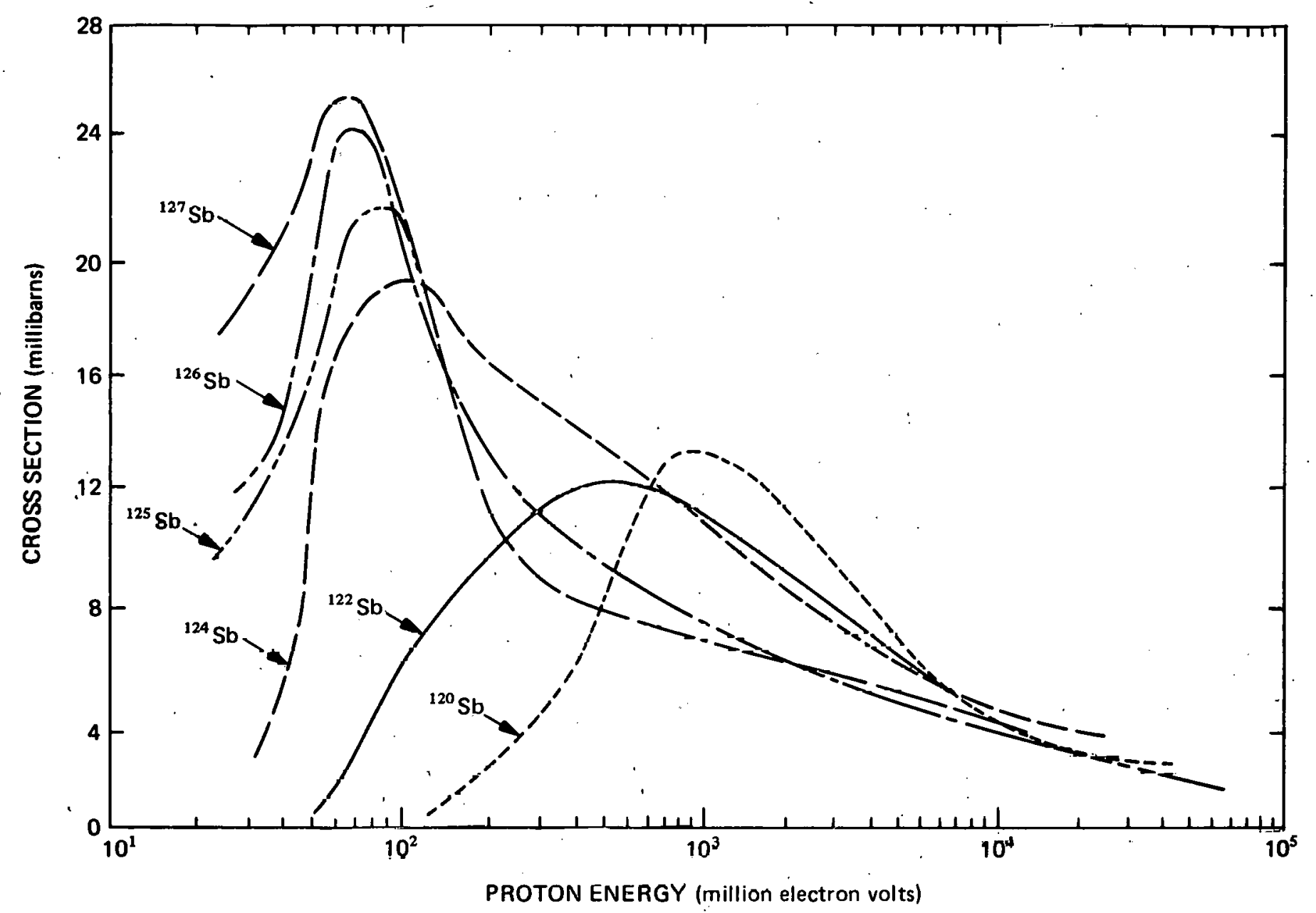

FIGURE 1. Excitation functions of antimony $(\mathrm{Sb})$ isotopes formed in the fission of uranium-238 by protons. Data above 160 million electron volts are those of Panontin and Sugarman (Reference 2.7) at 450 million elootron volts aind IIagel $\phi$ (Reterence 20 ) at 570 million electrion volts and 18.2 gigaclectron volls.

$$
\begin{aligned}
& \mathrm{Z} \text { - charge } \\
& \mathrm{Z}_{\mathrm{p}} \text { - most probable charge }
\end{aligned}
$$

When the logarithm of Equation 1 is taken, the expression assumes the form of a.parabola:

$$
\log P(Z)=-\frac{1}{c}\left(Z-Z_{p}\right)^{2}+\left(\log \frac{1}{\sqrt{c \pi}}\right)
$$

In the present work, the parameters are cross sections and units of $N / Z$. The use of the former implies a flat-mass yield curve, an approximation at the lower energies, but certainly correct for this Inass region at the higher energies. Equation 2 was expanded to the form of a second-order polynomial for fitting by the least-squares routine. Dispersion curves were also constructed from the data of Panontin and Sugarman (22) and Hageb $\phi$ (20). These are discussed later.

\section{DISCUSSION}

\section{Excitation Functions:}

The excitation functions, shown in Figure 1, of the independent-formed nuclides $\left({ }^{120} \mathrm{Sb},{ }^{122} \mathrm{Sb},{ }^{124} \mathrm{Sb}\right.$, ${ }^{126} \mathrm{Sb}$, and ${ }^{127} \mathrm{Sb}$ ) show the behavior expected of fission products formed at these energies. The data of Panontin and Sugarman (22) at $450 \mathrm{MeV}$ and of Hageb $\phi(20)$ at $570 \mathrm{MeV}$ and $18.2 \mathrm{GeV}$ bombarding energies have been included in order to obtain the energies at which the excitatiun functions reach their maxima.

The excitation functions of the two most neutrondeficient isotopes studied $\left({ }^{120} \mathrm{Sb}\right.$ and $\left.{ }^{122} \mathrm{Sb}\right)$ reach their peaks (interpolated) at about $1 \mathrm{GeV}$. The excitation functions for the independent formation 


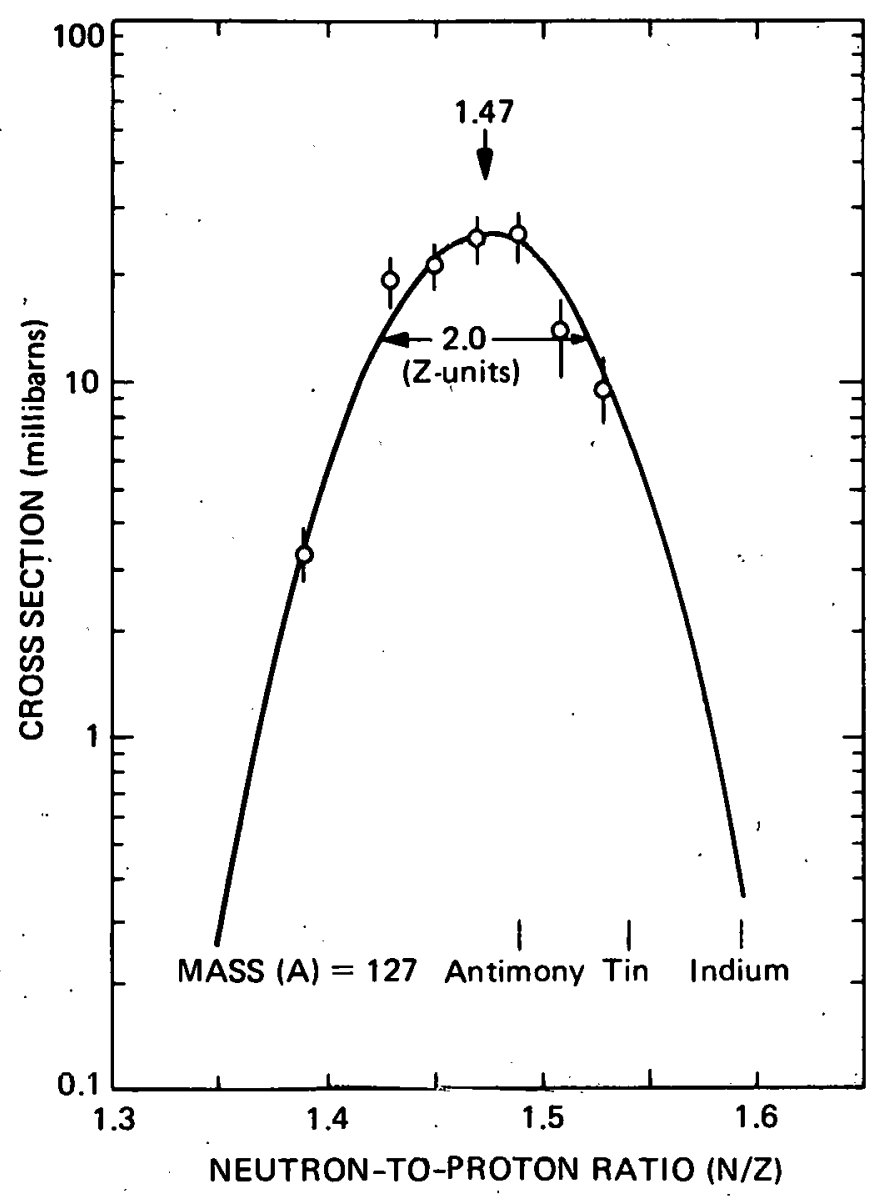

FIGURE 2. Typical charge-dispersion curve, obtained at 70 million electron volts bombarding energy, constructed by least-squares fitting of a Gaussian to data points. The yield for antimony$128 \mathrm{~g}$ has been added to obtain its isomer ratio.

of ${ }^{124} \mathrm{Sb},{ }^{125} \mathrm{Sb},{ }^{126} \mathrm{Sb}$, and ${ }^{127} \mathrm{Sb}$ show welldefined peaks within the present energy range studied. The functions clearly indicate also the decrease in energy at which the formation of the nuclide is most probable as the value of $\mathrm{N} / \mathrm{Z}$ increases. The excitation function for ${ }^{128} \mathrm{Sb}$ and those for ${ }^{129} \mathrm{Sb}$ and ${ }^{131} \mathrm{Sb}$ (actually cumulative, but close to independent because of their early position in the decay chain) reach their maxima at energies below those studied here.

Friedlander et al. (3) have shown if one plots the energies at which the excitation functions of cesiurn (Cs) isotopes reached their maxima versus the value of $N / Z$ of the nuclide that these values fall on a smooth curve. The data for rubidium- $84\left({ }^{84} \mathrm{Rb}\right)$ and ${ }^{86} \mathrm{Rb}$ fall on the curve as well. Subsequently the curve was extended to lower energies by Davies and Yaffe (5). Parikh et al. (6) found that data for barium, lanthanum, and cesium isotopes could be fitted on the curve. The finding was not surprising, since the mass ranges covered did not differ greatly from those of the cesium region.

It is of interest then to note that the data for the antimony isotopes, as shown in Figure 4 fit on the curve drawn from the previous data (6), even though the difference in mass is 10 to 20 units. The position of the antimony points shows that the curve reported by Davies and Yaffe (5) may not be linear at low energies. A forthcoming paper (23) will cover this aspect.

\section{Charge Dispersion:}

In Table IV are shown the parameters for the chargedistribution curves. As noted, in this energy range and mass region, correction for the variation of $\mathrm{N} / \mathrm{Z}_{\mathrm{p}}$ as a function of $\mathrm{A}$ is small. Thus Table IV contains the uncorrected data. Figure 5 shows the variation of $Z_{A}-Z_{p}$ as a function of energy, values of $Z_{A}$ being those of Coryell (24). The rapid initial drop, followed by a gradual movement towards stability, as found in other systems, is evident here.

The values of the full-widths at half-maximum increase from 1.6 to 2.5 charge units in the proton energy region: $E_{p}=30$ to $160 \mathrm{MeV}$. In order to determine the effect of the slope of the mass-yield curve on the width of the charge-dispersion curve, the cross-section data were converted to fractional yields, by dividing by the mass yields interpolated from the curves of Stevenson et al. (25). These cumulative yields need not be correct on an absolute scale, as long as they agree relatively, so that the slope is not in error. The yield curves (25) shuw the greatest slope in this mass region at a bombarding energy of $30 \mathrm{MeV}$. The widths of the chargedispersion curves increased only slightly when the parabolae were fitted to the fractional chain yields, showing that the assumption of a flat mass-yield curve in establishing the curves, even in the area of greatest change, scarcely perturbed the data. 


\section{RFP-2062}

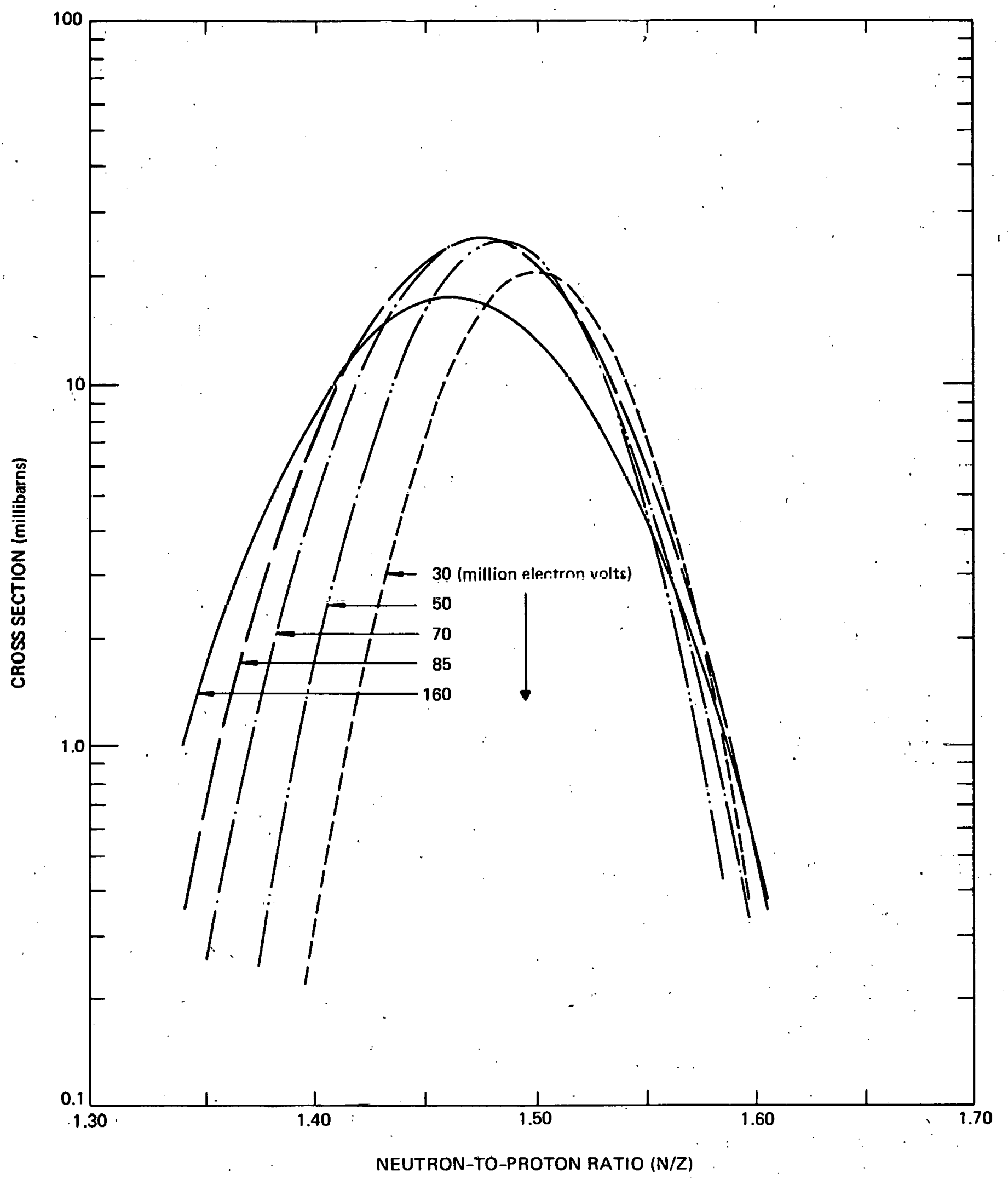

FIGURE 3. Charge-dispersion curves at 30 to 160 million electron volts. 


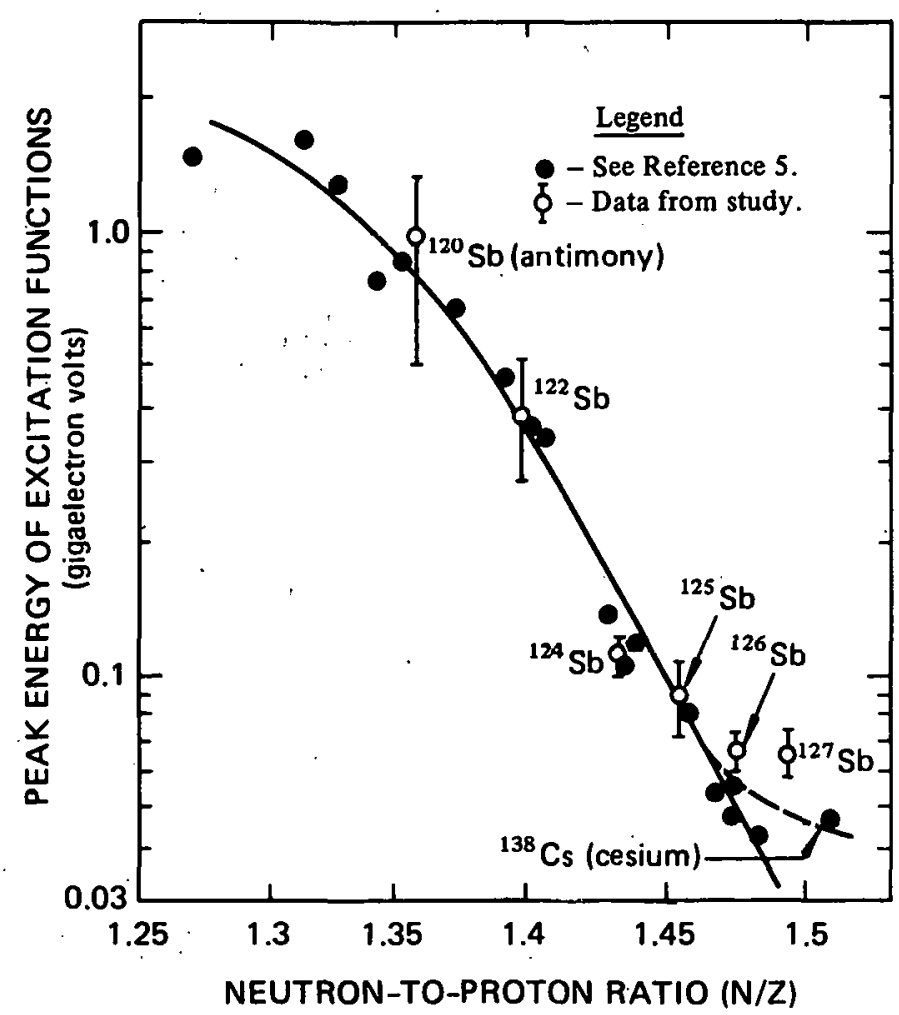

FIGURE 4. Energies at which excitation functions (See Figure 1) reach their maxima as a function of the value of the neutron to proton ratio $(\mathrm{N} / \mathrm{Z})$ of the fission product.
Hogan and Sugarman (4) have shown that in order to obtain correct values of FWHM for a chargedispersion curve, obtained from isotopic rather than isobaric data, the values must be corrected for the variation of $N / Z_{p}$ with $A$. It has been shown (26) at higher energies that, in the mass region presently under study, the value of $N / Z_{p}$ depends strongly on mass. The magnitude of this correction was found using data from previous studies $(5,6,7)$ covering mass ranges 90 to 93,130 to 138 , and 139 to 143, as shown in Figure 6. Also shown for comparison is the variation of $N / Z_{p}$ with $A$ for thermal-neutron fission (1) of ${ }^{235} \mathrm{U}$ and the fission of ${ }^{238} \mathrm{U}$ by $160-\mathrm{MeV}$ protons (26). These curves were used to calculate the value of $N / Z-N / Z_{p}$ for each isotope of antimony and the chargedispersion parameters were recalculated. The results, shown in Table $\mathrm{V}$ indicate that the isotopic curves are 10 to 19 percent wider than the isobaric curves in this mass and energy region.

Previous workers have used Gaussian or roughly symmetrical curves. Examination of the curve shown in Figure 2 indicates that a more flat-topped curve might also be compatible with the data.

TABLE IV. Parameters of Charge-Dispersion Curves.

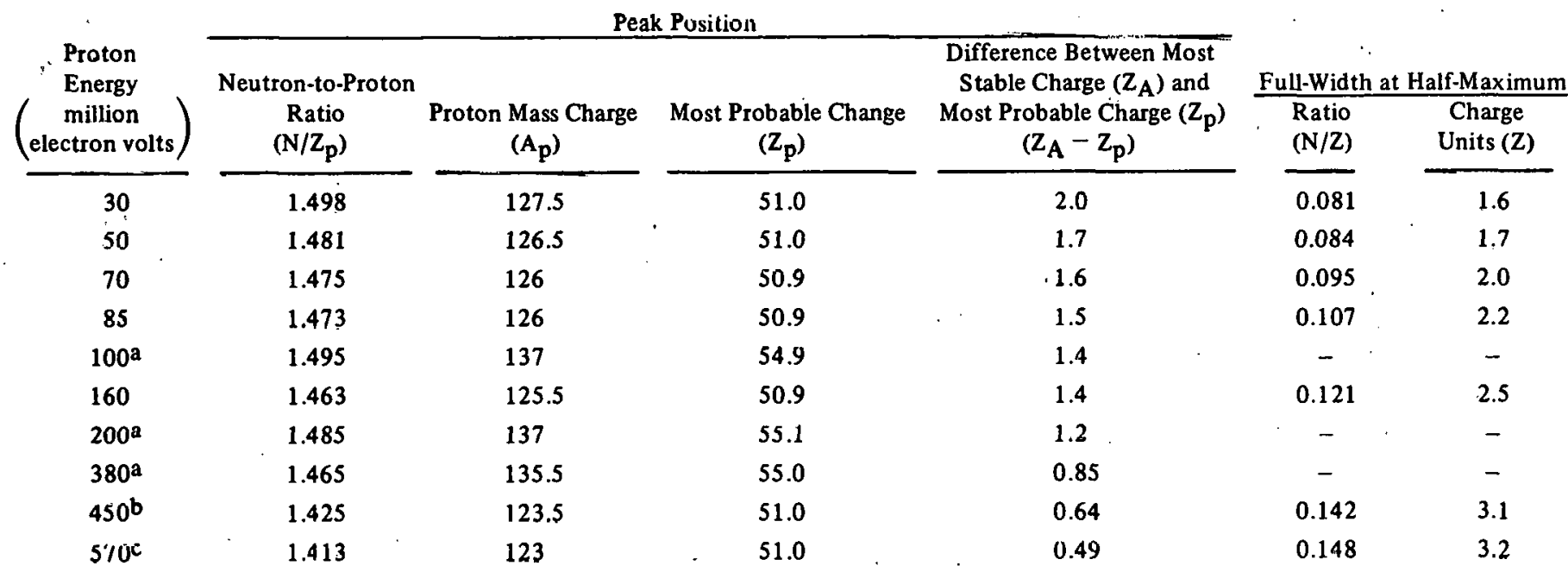

asee Reference 3.

bSee Reference 22.

cSee Reference 20. 


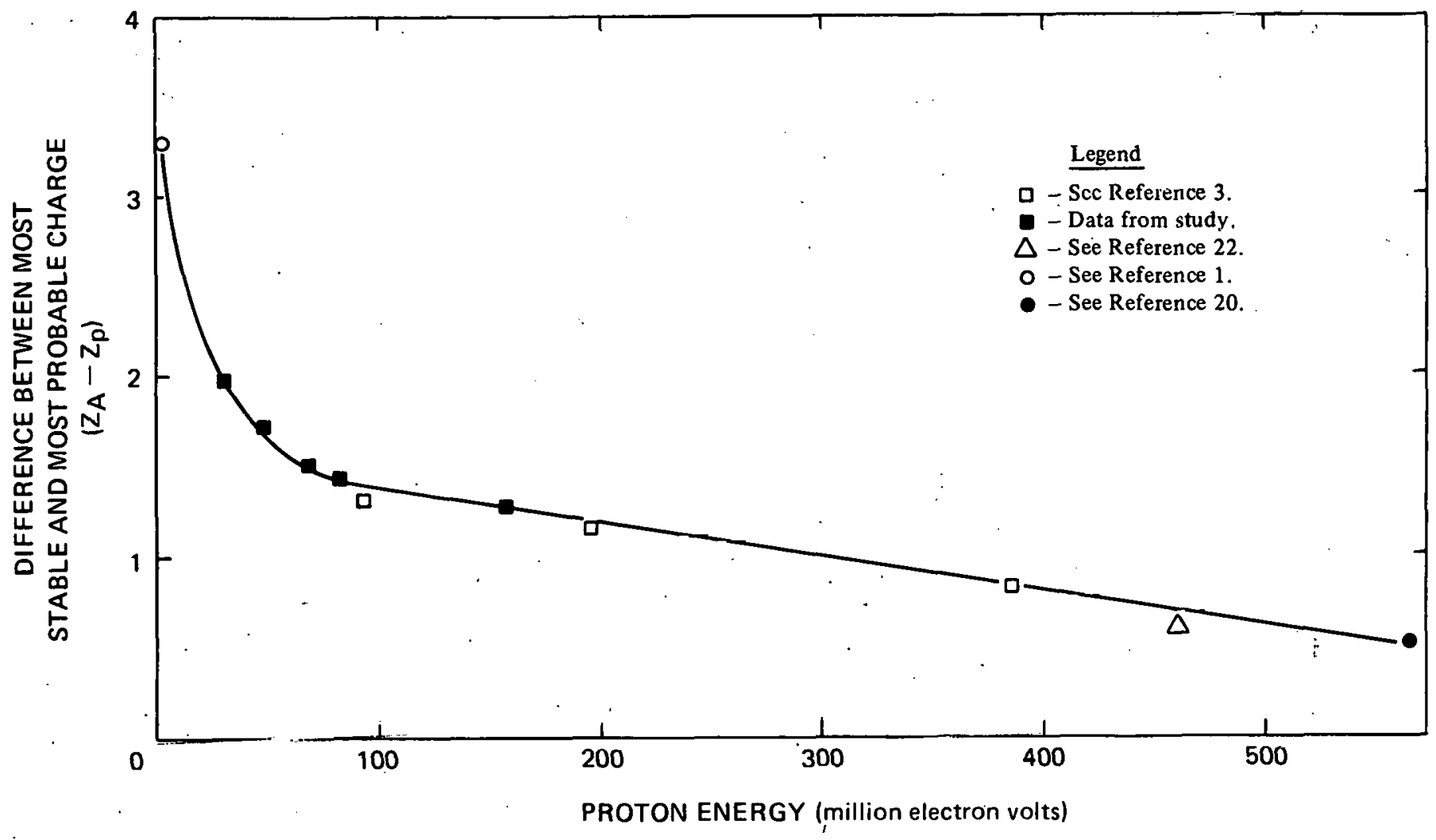

FIGUKE 3. Variation of the difference between most stable charge and most probable charge. $\left(Z_{A}-Z_{p}\right)$ as a function of bombarding energy.

Attempts were made to produce such a curvc by fitting twu uverlapping Gaussians by a computcr program to the experimental points. In all cases, exccpt for $30 \mathrm{MeV}$, the chi-square $\left(\chi^{2}\right)$ test showed significant improvement for the fit of the double curve over that of the single. The resulting curves are clearly asymmetrical, as shown for example in Figure 7 for the data at $85 \mathrm{MeV}$. The widths of the asymmetrical charge-dispersion curves, obtaincd in this fashion, were converted to $\mathrm{N} / \mathrm{Z}$ units, corrected for $\mathrm{N} / \mathrm{Z}-\mathrm{N} / \mathrm{Z}_{\mathrm{p}}$, as read from the second abscissa, (see Figure 7 and Table VI). Again a correction of about 20 percent is evident. The curve at $30 \mathrm{MeV}$ was not appreciably changed either by mass-yield corrections (a broadening of about $0.1 \mathrm{Z}$ units, well within experimental error) or by fitting to an asymmetric curve, but was made narrower by the correction of $N / Z_{p}$ with $A$.

McHugh and Michel (27) found their isobaric charge distribution curves to be Gaussian, constant in width to $39 \mathrm{MeV}$, the last being similar to the resulte of Patc et al. (2), and others. Indeed even the charge-dispersion curve in this work at $50 \mathrm{MeV}$ $\left(\mathrm{I}^{*}=52 \mathrm{MeV}\right)$ shows only slight asymmetry.

The widths of the charge-dispersion curves observed in the proton fission as a function of energy in the fission of uranium are shown in Figure 8. As apparent from the preceding discussion, none of the proposed corrections will cxplain the differences in the widths observed in the different mass regions at these energies. Figure 9 shows the variation of the FWHM at energies of 30 and $85 \mathrm{MeV}$. The dashed portion of the curves is an interpolation, the data currently being obtained at the McGill University Laboratory. The dependence of widths of the charge-dispersion curves upon mass in thermal-neutron fission is inconclusive. Wahl et al. . (1) assume a constant width for all mass chains,

\footnotetext{
*Excitation energies.
} 


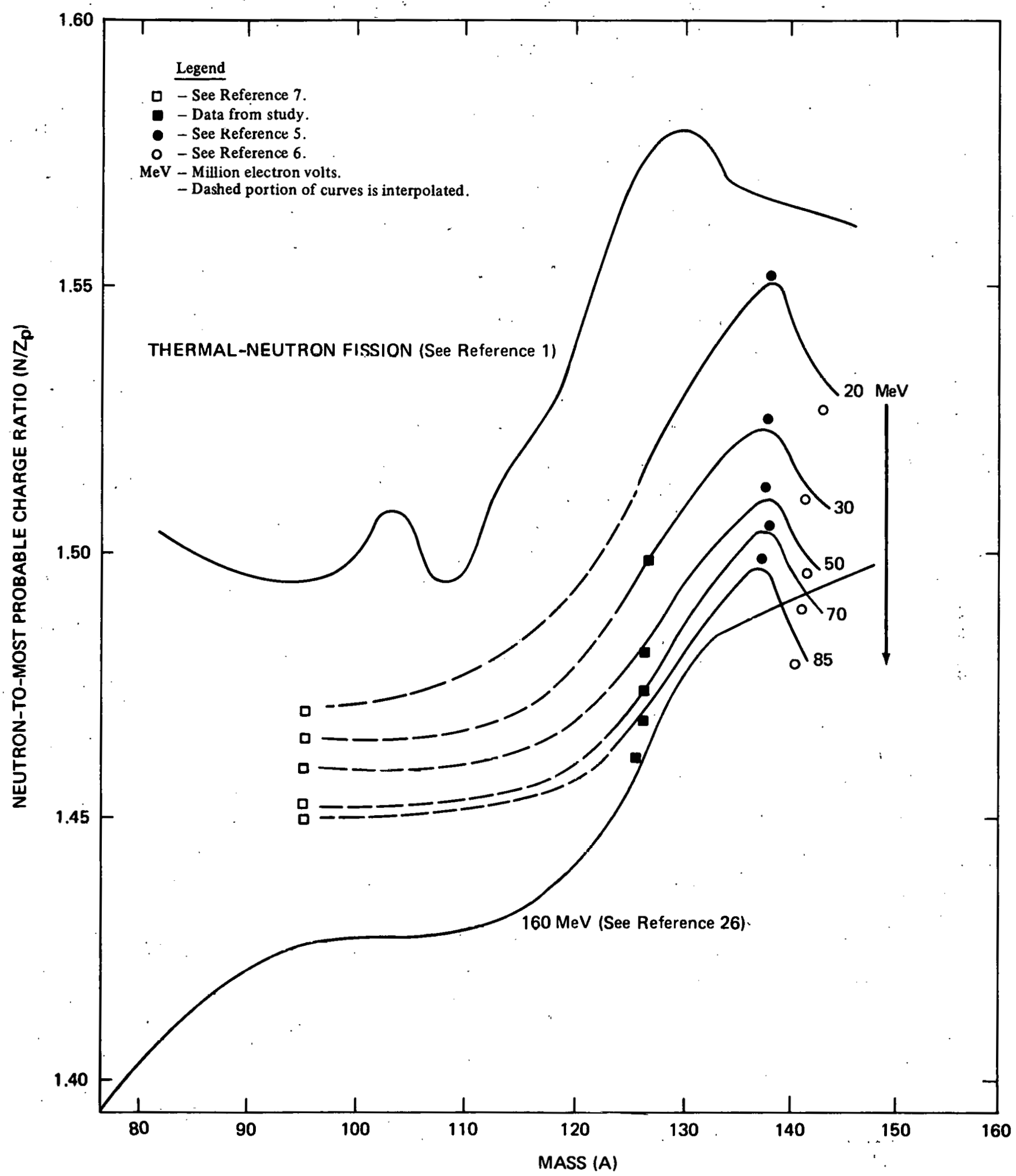

FIGURE 6. Variation of neutron-to-probable charge $\left(N / Z_{p}\right)$ with mass $A$ as a function of bombarding energy. 


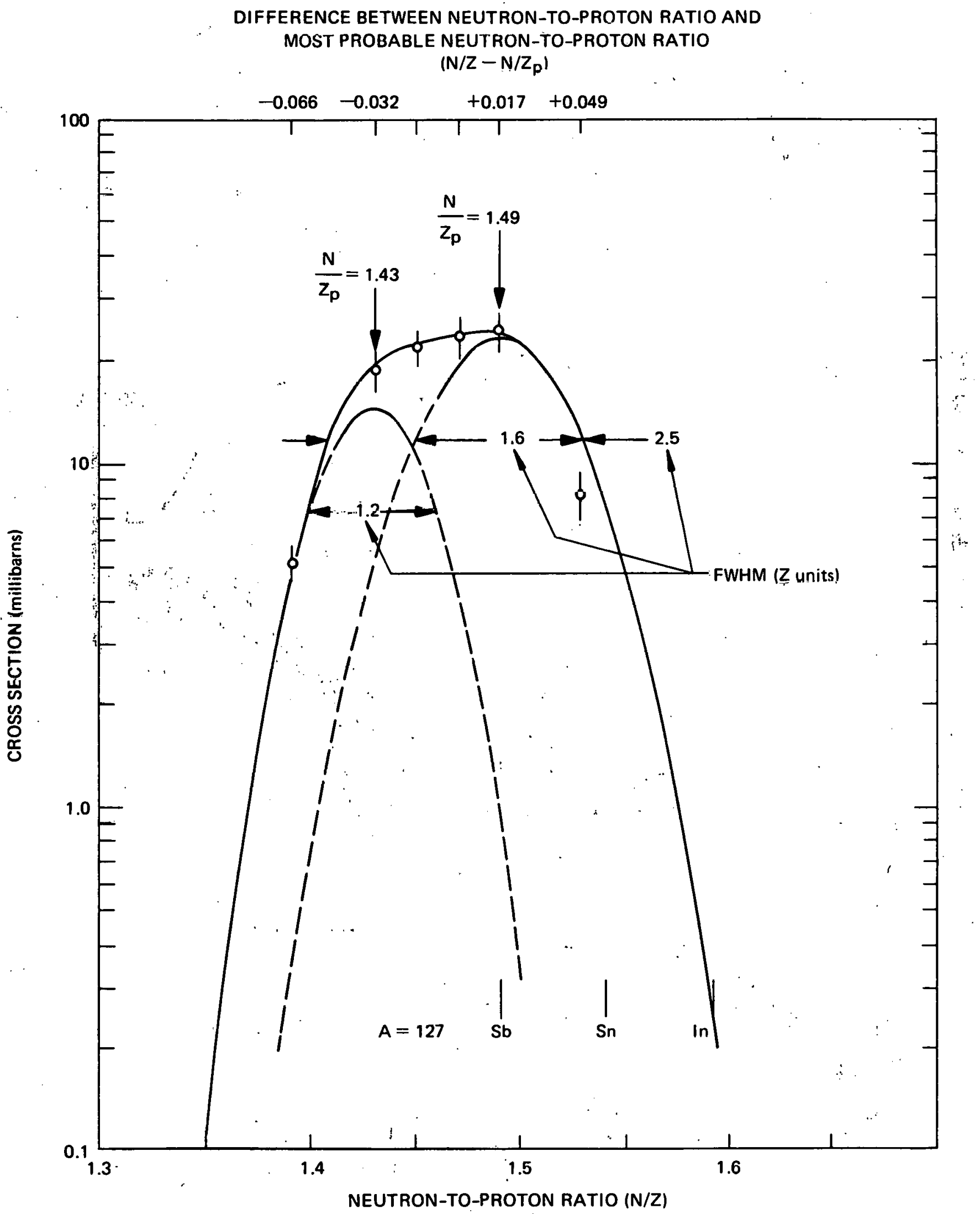

FIGURF 7. Charge-dispersion curve at 85 million electron volts fitted to two overlapping Gaussians. (Dashed portion of curves is interpolated.) 
TABLE V. Full-Width at Half-Maximum Value Corrected for Variation of Most Probable Neutronto-Proton Ratio with $\operatorname{Mass}(\mathrm{A}),\left[\mathrm{N} / \mathbf{Z}_{\mathrm{p}}(\mathrm{A})\right]$.

\begin{tabular}{|c|c|c|}
\hline $\left.\begin{array}{c}\text { Proton } \\
\text { Energy } \\
\text { million } \\
\text { electron volts }\end{array}\right)$ & $\begin{array}{c}\text { Uncorrected } \\
\text { Width } \\
\text { [charge units }(\mathrm{Z})]\end{array}$ & $\begin{array}{c}\text { Corrected for } \\
\mathrm{N} / \mathrm{Z}_{\mathrm{p}}(\mathrm{A}) \\
\text { [charge units (Z) }\end{array}$ \\
\hline 30 & 1.7 & 1.5 \\
\hline 50 & 1.7 & 1.5 \\
\hline 70 & 2.0 & 1.7 \\
\hline 85 & 2.2 & 2.0 \\
\hline \multirow[t]{2}{*}{160} & 2.5 & 2.1 \\
\hline & $\begin{array}{l}\text {-most probable cha } \\
\text { vith mass. } \\
\text { ic charge dispersion }\end{array}$ & tio $\left(N / Z_{p}\right)$ \\
\hline
\end{tabular}

TABLE VI. Corrected Charge-Dispersion Parameters.

\begin{tabular}{|c|c|c|c|c|c|}
\hline \multirow{3}{*}{$\left.\begin{array}{l}\text { Proton } \\
\text { Energy } \\
\text { (million } \\
\text { electron } \\
\text { volts }\end{array}\right)$} & & & \multicolumn{3}{|c|}{ Full-Width at Half-Maximum } \\
\hline & \multicolumn{2}{|c|}{ Peak Position } & & & correction \\
\hline & $\left(\mathbf{N} / \mathbf{Z}_{\mathrm{p}}\right)$ & $\left(Z_{A}-Z_{p}\right)$ & $(\mathrm{N} / \mathrm{Z})$ & (Z-Units) & applied \\
\hline 30 & 1.498 & 1.96 & 0.071 & 1.5 & $\mathbf{a}$ \\
\hline 50 & 1.481 & 1.68 & 0.081 & 1.7 & $a, b$ \\
\hline 70 & 1.475 & 1.59 & 0.10 & 2.1 & $a, b$ \\
\hline 85 & 1.473 & 1.55 & 0.10 & 2.1 & $\mathbf{a , b}$ \\
\hline 160 & 1.463 & 1.37 & 0.116 & 2.4 & $\mathbf{a , b}$ \\
\hline
\end{tabular}

while Notea (28), and Strom et al. (29) observed a small variation in width.

\section{CONCLUSION}

The filting of two Gaussians to the chargedispersion data tempts one to interpret the results in terms of two discretc processes, e.g., symmetric and asymmetric fission. Galin et al. (30) in the fission of ${ }^{238} \mathrm{U}$ by $155-\mathrm{MeV}$ protons concluded that 30 percent of the fissions of uranium are asymmetric, while 70 percent are symmetric. At mass 125 , the results of Galin et al. indicate that the ratio asymmetric to symmetric is 0.2 . Symmetric fission should contribute more to the light isotopes of antimony than to the heavy isotopes.
TABLE VII. Relative A reas of the Right-Hand Gaussian to the Left-Hand Gaussian as a Function of Energy.

\begin{tabular}{c}
$\begin{array}{c}\text { Proton Energy } \\
\text { (million electron volts) }\end{array}$ \\
\hline 50 \\
70 \\
85 \\
160
\end{tabular}

Ratio (area of heavy-mass Gaussian) (area of light-mass Gaussian)

7.9

2.4

2.0

1.5
However, as noted from Table VII, this is not the case. If the ratios of the heavy to the light-mass Gaussians are taken as indicative of the relative contributions from asymmetric and symmetric fission respectively, then at $30 \mathrm{MeV}$ the relative symmetric contribution is small, but increases in importance, until the two are nearly equal at $160 \mathrm{MeV}$.

If the data of Amarel et al. (31) were considered for cesium isotopes ( $A=128$ to 144), formed in the fission of ${ }^{238} \mathrm{U}$ by $150-\mathrm{MeV}$ protons, and were treated in similar manner, using fractional yields because of the inconstant mass yield in this mass region, the implied ratio of symmetric to asymmetric fission would be 1.2 [in good agreement with the value of 1.0 reported by Galin et ul. (30) for this region]. 


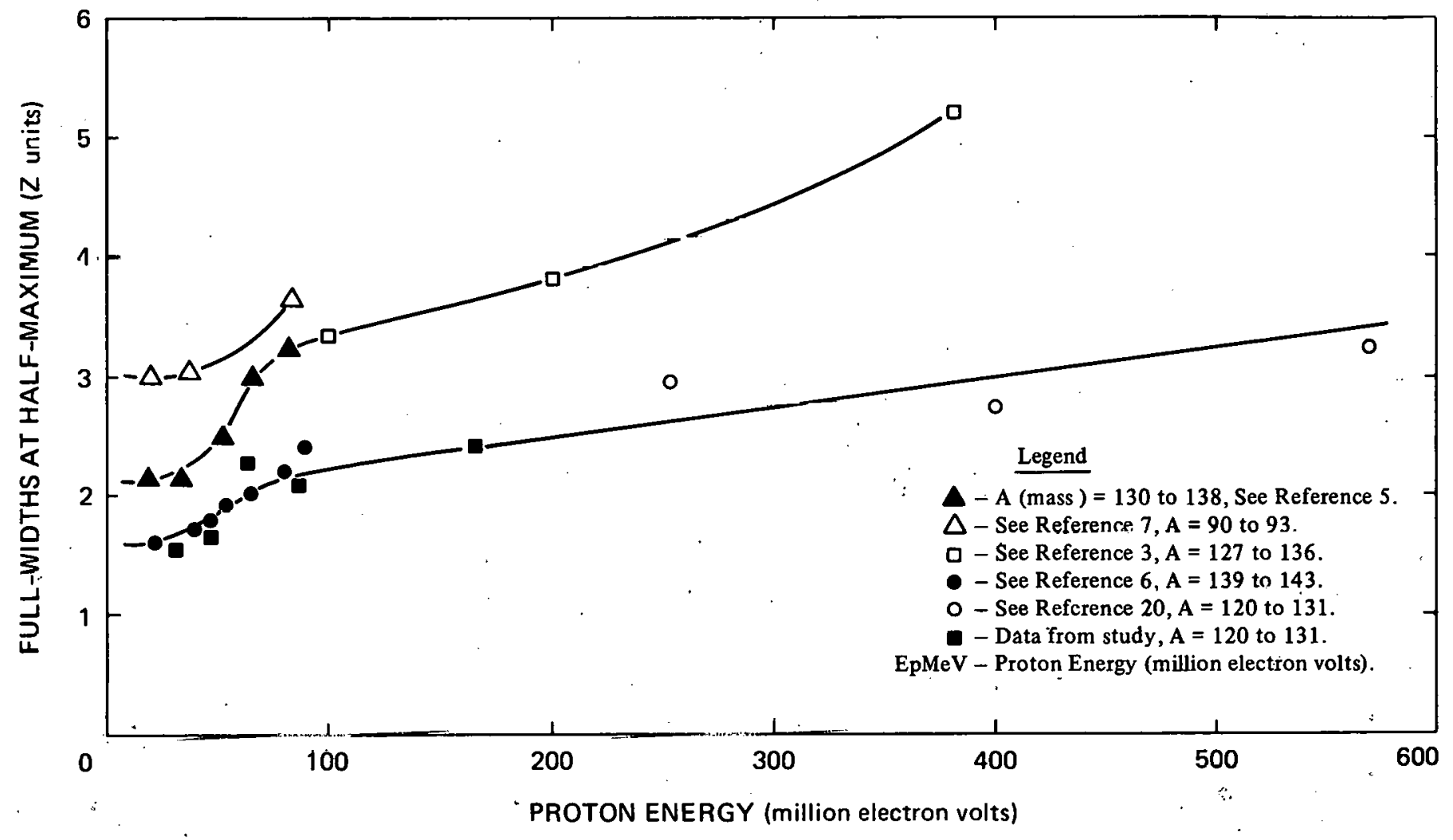

FIGURE 8. Dependence of full-width at half-maximum of the charge-dispersion curves on bombarding energy.

FIGURE 9. Variation of the full-width at half-maximum of oharge-disperslun cuives as a funcliun uf mass of the fission product at 30 and 85 million electron volts bombarding energy.

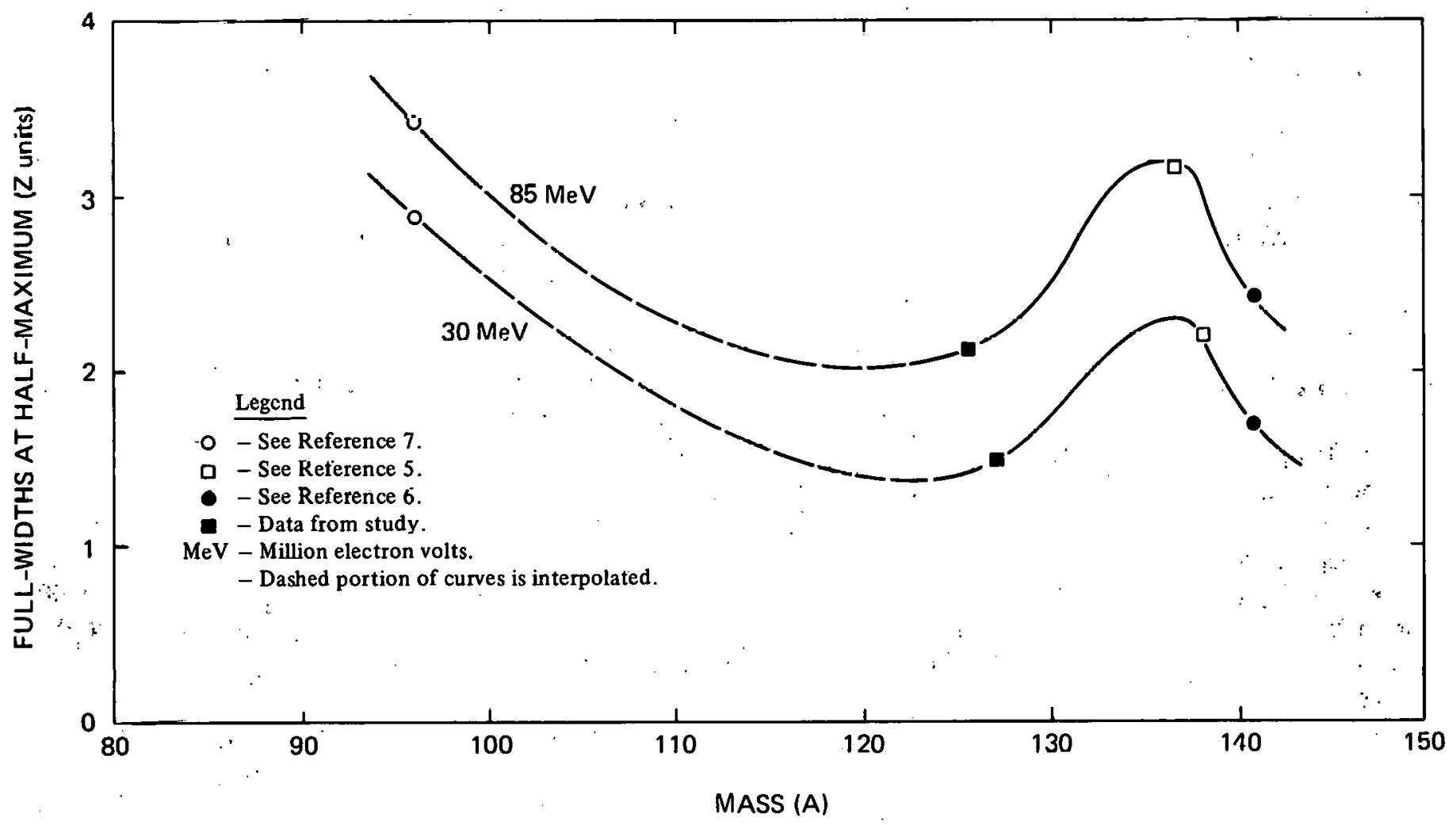




\section{REFERENCES}

1. A. C. Wahl, A. E. Norris, R. A. Rouse, and J. C. Williams. "Products from ThermalNeutron-Induced Fission of ${ }^{235}$ U." Physics and Chemistry of Fission. Paper SM-122/166. International Atomic Energy Agency. Vienna, Austria. 1969.

2. B. D. Pate, J. S. Foster and L. Yaffe. "Distribution of Nuclear Charge in the ProtonInduced Fission of ${ }^{232}$ Th." Canadian Journal of Chemistry, 36:1961. 1958.

3. G. Friedlander, L. Friedman, B, Gordon, and L. Yaffe. "Excitation Function and Nuclear Charge Dispersion in the Fission of Uranium by 0.1 - to $6.2-\mathrm{GeV}$ Protons." Physics Review, 129:1809. 1963.

4. J. J. Hogan and N. Sugarman. "Production of Heavy Nuclides in 440-MeV Proton Fission of Uranium." Physics Review, 182:1210. 1969.

5. J. H. Davies and L. Yaffe. "Nuclear Charge Dispersion in the High-Energy Fission of Uranium." Canadian Journal of Physics. $41: 762.1963$.

6. S. S. Parikh, D. A. Marsden, N. T. Porile, and L. Yaffe. "Isobaric Yield Distribution in the Fission of Uranium by Medium-Energy Protons." Canadian Journal of Chemistry, 45:1863. 1967.

7. A. H. Khan, G. B. Saha, and L. Yaffe. "Nuclear Charge Dispersion of Light-Mass Fission Products in the Fission of ${ }^{235} \mathrm{U}$ and ${ }^{238} \mathrm{U}$ by Medium-Energy Protons." Canadian Journal of Chemistry, 48:1924. 1970.
8. G. B. Saha and L. Yaffe. "Charge Dispersion Studies in the Fission of ${ }^{239} \mathrm{Pu}$ by Protons of Medium Energy." Journal of Inorganic Nuclear Chemistry, 32:745. 1970.

9. I. Tomita and L. Yaffe. "Nuclear Charge Dispersion in the Fission of ${ }^{233} \mathrm{U}$ by Protons of Medium Energy." Canadian Journal of Chemistry, 47:2921. 1969.

10. G. B. Saha, I. Tomita, and L. Yaffe. "Nuclear Charge Dispersion in the Fission of ${ }^{235} \mathrm{U}$ by Protons of Energy 20-85 MeV.". Canadian Journal of Chemistry, 49:2205. 1.971.

11. P. P. Benjamin, D. A. Marsden, N. T. Porile, and L. Yaffe. "Nuclear Charge Dispersion in the Fission of ${ }^{232} \mathrm{Th}$ by Protons of Energy 20-85 MeV." Canadian Journal of Chemistry, 47:301. 1969.

12. T. McGee, C. L. Rao, and L. Yaffe. "Nuclear Charge Dispersion in the Fission of ${ }^{237} \mathrm{Nb}$ by Protons of Medium Energy." Journal of Inorganic Nuclear Chemistry, 34:3323. 1972.

13. L. Yaffe. "Nuclear Charge Dispersion in the Fission of Heavy Elements by Medium-Energy Protons.". Physics and Chemistry of Fission, Paper SM-122/3. International Atomic Energy Agency, Vienna, Austria. 1969.

14. A. C. Pappas and E. Hageb $\phi$. "The Charge and Mass Distributions in Fission of Uranium Induced by $170 \mathrm{MeV}$ Protons." Journal of Inorganic Nuclear Chemistry, 28:1769. 1966.

15. A. E. Greendale and D. C. Love. "Rapid Radiochemical Procedure for Antimony and Arsenic." Analytical Chemistry, 35:632. 1963. 
16. W. H. Latimer and J. H. Hildebrand. Reference Book of Inorganic Chemistry. The MacMillan Company, New York. 1951. Page 241.

17. K. A. Kraus and G. E. Moore. Journal of the American Chemical Society, 75:1460. 1953.

18. S. Meghir: PhD Thesis. Department of Chemistry, McGill University, Montreal, Canada. 1962.

19. J. B. Cumming. "Monitor Reactions for High Energy Proton Beams." Annual Review of Nuclear Science, 13:261. 1963.

20. E. Hage $b \phi$. "Yields and Isomeric Yields Ratios of Antimony Isotopes from the Interaction of $159 \mathrm{MeV}$ to $18.2 \mathrm{GeV}$ Protons with Uranium." Journal of Inorganic Nuclear Chemistr, 2.9.2515. 1967.

21. N. K. Aras and G. E. Gordon." The Fractioned Chain Yield of ${ }^{126} \mathrm{Sb}$ in ThermalNeutron Fission of ${ }^{235}$ U." Journal of Inorganic Nuclear Chemistry, 29:763. 1966.

22. J. A. Panontin and N. Sugarman. "Mass Yield Distribution and Charge Dispersion in $450 \mathrm{MeV}$ Proton Fission of ${ }^{238}$ U:" Journal of Inorganic Nuolcar Chemisiry, 34:1485. 19'/2.

23. R. Holub and L. Yaffe. Private Communication. June 1970.

24. C. D. Coryell. " $\beta$-Decay Energetics." Annual Review of Nuclear Science, 2:305. 1953.

25. P. C. Stevenson, H. G. Hicks, W. E. Nervik, and D. R. Nethaway. "Further Radiochemical Studies of the High-Energy Fission Products." Physics Review, 111:886. 1958.

26. A. C. Pappas and E. Hageb $\phi$. Loc. cil.

27. J. A. McHugh and M. C. Michel. "FissionFragment Mass and Charge Distribution for the Moderately Excited ${ }^{236} \mathrm{U}$ Compound Nucleus." Physics Review, 172:1160. 1968.
28. A. Notea. "Distribution of Nuclear Charge in Low-Energy Fission of Transthorium Elements." Physic's Review, 182:1331. 1969.

29. P. O. Strom, D. L. Love, A. E. Greendale, A. A. Delucchi, D. Sam, and N. F. Ballou. "Nuclear-Charge Distribution of FissionProduct Chains of Mass Numbers 131-133." Physics Review, 144:984. 1966.

30. J. Galin, M. Lefort, J. Péter, X. Tarrago, E. Cheifetz, and Z. Fraenkel. "Etude Compare'e de la Fission du Bismuth èt de l'Uranium Induite par des Protons de Grande Energie (156 MeV)." Nuclear Physics, A134:513. 1969 .

31. I. Amarel, R.Bernas, J. Chaumont, R. Houcher, J. Jastrzebski, A. Johnson, R. Klapisch; and J. Teillac. Arkiv für Fysik, 36:77. 1966.

32. C. M. Lcderer, J. M. Hullander, and I. Perlman. Table of Isotopes. Sixth Edition. John Wiley and Sons, New York. 1967.

33. R. Gunnick, J. B. Niday, R. P. Anderson, and R. A. Meyer. UCID-15439. University of California. Lawrence Radiation Laboratory, Berkeley, California. 1.969.

34. C. J. Orth, B. J. Dropesky, and N. J. Freeman. "Decay of ${ }^{126} \mathrm{Sn}$ and the 19-min and 12.4-day Isomcrs of ${ }^{126} \mathrm{Sb} . "$ Physics Review, C3:2402. 1971.

35. P. G. Calway and H. D. Sharma. "The Excited States of ${ }^{129} \mathrm{Te}$ Populated in the $\beta^{-}$Decay of ${ }^{129}$ Sb." Nuclear Physics, A1 56:338. 1970.

36. B. J. Dropesky and C. J. Orth. "A Summary of the Decay of Some Fission Product Tin and Antimony Isotopcs." Journal of Inorganic and Nuclear Chemistry, 24:1301. 1962. 